\title{
Modelling and Numerical Enhancements on a UVLM for Nonlinear Aeroelastic Simulation
}

\author{
Stefanie Düssler*, Norberto Goizueta ${ }^{\dagger}$, Arturo Muñoz-Simón ${ }^{\ddagger}$ and Rafael Palacios ${ }^{\S}$ \\ Department of Aeronautics, Imperial College London, SW7 2AZ
}

\begin{abstract}
This work presents various improvements made to the Unsteady Vortex Lattice Method (UVLM) integrated into the open-source, nonlinear aeroelastic simulation environment SHARPy. The UVLM is extended by non-lifting body effects, polar corrections, and a new wake discretization scheme. The theory behind these enhancements is discussed and successfully verified. Finally, some of these enhancements are employed on a flexible aircraft demonstrator model. The results indicate an influence of the fuselage on the aeroelastic behavior of the wing, which becomes increasingly important for larger wing deformations and fuselage diameters. The polar corrections provide valuable refinements to the aerodynamic forces and moments, and the new wake discretization scheme significantly speeds up the simulations.
\end{abstract}

\section{Introduction}

$\mathrm{T}$ HE unsteady vortex lattice method (UVLM) is a medium-fidelity tool to predict non-stationary aerodynamic loads. Despite the limitations of potential flow theory, the UVLM has proved to be a remarkable tool, especially when it comes to unsteady aerodynamics in vehicles with coupled aeroelasticity and flight dynamics. For example, Goizueta et al. [? ] have recently obtained excellent flutter prediction for a very flexible wing and demonstrated the advantage of UVLM for applications with large wing deformations. Del Carre and Palacios [? ] have successfully optimized the complex maneuvers of very flexible aircraft using the UVLM. Deskos et al. [? ] have assessed various turbulence models for aircraft aeroelasticity and have even been capable of inputting large LES data into the UVLM at affordable computational costs.

These research projects have been carried out using SHARPy, our open-source, nonlinear aeroelastic simulation environment [? ]. It couples structural dynamics captured with a nonlinear geometrically-exact composite beam model (GEBM) with unsteady aerodynamics solved with the unsteady vortex lattice method (UVLM). This paper reports on some recent improvements to the UVLM by tackling some of its previous limitations. In particular, we here introduce non-lifting body effects and viscous drag corrections and speeding up the numerical performance by a new proposed wake discretization scheme.

The effects of non-lifting bodies cannot be captured by the UVLM since it is only applicable to thin, lifting surfaces. Therefore, SHARPy's UVLM module is extended in the present paper by a linear source panel method with a mathematical formulation based on the derivation made by Hess and Smith [? ]. Then, the UVLM and the source panel method are coupled using superposition and a vortex strength correction is deployed at wing-body-junctions. A similar approach has been proposed by Wasserstrom et al. [? ]. This extension of SHARPy allows the simulation of wing-fuselage-configurations.

Another limitation of the UVLM is that it does not account for viscous drag [? ] since the UVLM is based on inviscid flow equations. Viscous drag is, however, essential for the correct estimation of power production of wind turbines [? ], the efficiency of aircraft, and affects as well flight dynamics (the phugoid mode in particular). Drag corrections based on the integral boundary layer equation and the transpiration velocity concept [? ] have been proposed in the past. In the present work, we propose a simple sectional drag correction in UVLM based on look-up tables obtained from either wind tunnel experiments or blade-resolved CFD, similar to the correction for lift proposed in [? ].

Furthermore, the UVLM has been improved regarding its numerical performance. The UVLM captures unsteady and three-dimensional effects on aerodynamic loads at the cost of a very demanding free wake discretisation and convection [? ? ]. For very dense and long wakes, as the ones generated behind wind turbines, the computational cost increases significantly [? ]. This cost can be reduced by transforming vortex rings into vortex blobs when they are far

\footnotetext{
*Graduate research assistant, Department of Aeronautics, s.dussler20@imperial.ac.uk

†raduate research assistant, Department of Aeronautics, norberto.goizueta13@imperial.ac.uk

${ }^{\ddagger}$ Graduate research assistant, Department of Aeronautics, a.munoz-simon@imperial.ac.uk

§Professor of Computational Aeroelasticity, Department of Aeronautics, r.palacios@imperial.ac.uk, AIAA Associate Fellow
} 
away from solid surfaces [? ? ] or using the fast multipole method [? ]. Here, we propose a new wake convection scheme that relaxes the wake discretisation requirements reducing the computational cost. In particular, any arbitrary wake shape is maintained as opposed to previous works that assume simple geometries [? ].

All the previous enhancements to the UVLM are discussed in this work. This document is organized such that we present in Section [I]the methodologies used in SHARPy as well as in the new proposed features, namely the non-lifting body model, the sectional drag correction and the wake discretization. Section III introduces verification results of the non the non-lifting body model and the sectional drag corrections. Subsequently in Section IV] we present the implementation of a demonstrator aircraft model along with the effect of our fuselage model and polar correction.

\section{Technical Background}

In this section, we first introduce the reader to the structural and aerodynamic methods that form the non-linear time-domain aeroelastic solver in SHARPy. Subsequently, we formulate physical models which extend the UVLM with non-lifting body effects and viscous drag effects. Finally, a numerical improvement to the UVLM in the form of a new proposed wake convection scheme is presented.

\section{A. Nonlinear time-domain aeroelastic solver}

\section{Nonlinear Geometrically-Exact Composite Beam Model}

The structural model of choice is a nonlinear geometrically-exact composite beam model (GEBM). The GEBM has linear constitutive relations and nonlinear velocity and displacement kinematic relations. The beam is discretized in quadratic (3-node) finite elements [? ? ] and parametrized in nodal displacements and rotations, denoted by $\boldsymbol{\eta}$, which are given in a body-attached, moving frame of reference (FoR) $A$. The $A$-frame moves and rotates with respect to an inertial frame, denoted by $G$ although in this work it will be stationary.

The beam dynamics are derived by applying Hamilton's principle [? ] and are expressed in its nonlinear formulation as

$$
\mathcal{M}(\boldsymbol{\eta}) \ddot{\boldsymbol{\eta}}+\boldsymbol{Q}_{\mathrm{gyr}}(\boldsymbol{\eta}, \dot{\boldsymbol{\eta}})+\boldsymbol{Q}_{\mathrm{stiff}}(\boldsymbol{\eta})=\boldsymbol{Q}_{\mathrm{ext}}(\boldsymbol{\eta}, \dot{\boldsymbol{\eta}}),
$$

where $\mathcal{M}$ is the mass matrix and the matrices denoted by $\boldsymbol{Q}$ include the gyroscopic, stiffness, and external generalised forces. The equation is solved iteratively with a Newton-Raphson scheme and an explicit, incrementally formulated Newmark- $\beta$ scheme performs the time integration [? ].

\section{Unsteady Vortex Lattice Method}

For fluid-structure interaction (FSI) problems, SHARPy couples the structural model at each FSI time step to the UVLM. The UVLM is based on potential flow and thin wing theory and provides a medium-fidelity tool for the prediction of unsteady aerodynamic loads for three-dimensional low-speed and attached flow over lifting surfaces [? ].

For the UVLM, the instantaneous geometry of the lifting surface $\zeta(t)$ is first discretized into a lattice of quadrilateral panels. The panels are coincident with the structural nodes and on each panel, a bound vortex ring (index b) with a circulation, denoted by $\boldsymbol{\Gamma}_{\mathrm{b}}$, is placed.

Second, another lattice of vortices with the circulation strengths $\boldsymbol{\Gamma}_{\mathrm{w}}$ is needed to capture the wake. The wake forms because of shed vortices that arise to fulfill the Kutta-Joukowski condition at the trailing edge and convect downstream [? ]. Several wake convection schemes to capture the shape of the wake exist, namely: fixed flat wake, prescribed wake, convected wake, and free wake (ordered by increasing fidelity). For steady computations, the prescribed wake is a sufficient approach for FSI simulations. Here, the wake is forced to be shed from the trailing edge, leading to a predetermined position of the wake vortex rings. For unsteady computations, however, more accurate results are obtained with the convected wake scheme in which the vortex rings convect with the background flow information, including atmospheric disturbances [? ].

The most accurate convection scheme is the free wake approach. This approach considers not only the background flow and atmospheric disturbances, but also the velocities induced by each vortex, enabling the capturing of wake-roll up effects. However, it should be noted that this approach involves considerable computational costs and in most cases the differences in the resulting aerodynamic loads are negligible compared to the convective (prescribed) wake.

After setting up the geometry and placing the singularity elements on the lifting surface and wake panels, the 
induced velocity field at an arbitrary point in space $\boldsymbol{x}$ can be calculated from

$$
\boldsymbol{u}(\boldsymbol{x}, t)=\mathcal{A}_{b}(\boldsymbol{x}, \zeta(t)) \boldsymbol{\Gamma}_{\boldsymbol{b}}(t)+\mathcal{A}_{w}(\boldsymbol{x}, \zeta(t)) \boldsymbol{\Gamma}_{\boldsymbol{w}}(t) .
$$

The aerodynamic influence coefficient (AIC) matrices $\mathcal{A}_{b}$ and $\mathcal{A}_{w}$ contain contain the normal velocity component $\boldsymbol{u}\left(\boldsymbol{x}_{i j}, t\right) \cdot \boldsymbol{n}_{j}$ on a collocation point, located at the center of panel $j$, induced by the vortex ring placed on panel $i$. This induced velocity of a vortex segment is calculated by Biot-Savart's law.

To calculate the unknown circulation strengths $\boldsymbol{\Gamma}_{\boldsymbol{b}}$ and $\boldsymbol{\Gamma}_{\boldsymbol{w}}$, we now enforce the non-penetrating boundary condition on the collocation points, which leads to the linear set of equations $\mathcal{A}_{l} \boldsymbol{\Gamma}_{l}=-\boldsymbol{B}_{l}$ with the boundary conditions $\boldsymbol{B}_{l}=\left(\boldsymbol{U}_{\infty}+\boldsymbol{U}_{\text {ext }}\right) \cdot \boldsymbol{n}$ and the AIC matrix $\mathcal{A}_{l}$. The solution to this linear set of equations finally gives the circulation strengths $\boldsymbol{\Gamma}_{l}$. The subindex $l$ denotes for the lifting surface and includes the combined bound $b$ and wake $w$ vertices. $\boldsymbol{U}_{\infty}$ is the freestream velocity vector and $\boldsymbol{U}_{\text {ext }}$ includes other velocity contributions as gust velocities.

From the obtained circulation strengths $\boldsymbol{\Gamma}_{l}$, we can finally compute the resulting aerodynamic forces using the unsteady Kutta-Joukowsky theorem [? ]. Vortex rings induce a quasi-steady force, considering leading edge suction effects, and an unsteady force, accounting for the added mass effect [? ]. Both force components contribute to the total resulting force $\boldsymbol{F}=\boldsymbol{F}_{\text {steady }}+\boldsymbol{F}_{\text {unsteady }}$. The steady force results for the UVLM in

$$
\boldsymbol{F}_{\text {steady }, s}=\rho_{\infty} \Gamma_{s}\left(\overline{\boldsymbol{v}}_{s} \times \boldsymbol{l}_{s}\right),
$$

where the subindex $s$ denotes the specific vortex segment, $\boldsymbol{l}$ the segment vector, and $\overline{\boldsymbol{v}}_{s}$ the relative segment-fluid velocity. The unsteady force for the $k$-th collocation point is given by

$$
\boldsymbol{F}_{\text {unsteady, }, k}=\rho_{\infty} S_{k} \boldsymbol{n}_{k} \dot{\boldsymbol{\Gamma}}_{k}
$$

with the panel area $S$ and the time derivative of the vortex circulation $\dot{\boldsymbol{\Gamma}}$. The described approach has proven to be very accurate in the calculation of low-speed aerodynamic loads, captures induced drag effects, and has good convergence properties [?].

\section{B. Non-Lifting Body Effects}

From a lift-generating point of view, the fuselage is rather unimportant for aerodynamic load calculations of the typical commercial wing-tube configurations. Its main purpose is to provide space for passengers and payloads and contains the cockpit and part of the fuel tanks [? , Chapter 4]. However, strong interference effects between wing and fuselage cause the forces on one body to be significantly influenced by the flow field of the other body [?, Chapter 1]. Additionally, the presence of the fuselage affects the flight dynamics, in particular the neutral point position, the pitching moment, and the roll stiffness.

To capture these effects, we extend our aerodynamic solver based on the UVLM method by the linear source panel method. The method is outlined in this section along with the coupling of this method with the UVLM for the steady and unsteady case.

\section{Linear Source Panel Method}

The aerodynamics of non-lifting bodies are modeled by the linear source panel method also based on potential flow theory, in which the wetted surface of the non-lifting body is again discretised into quadrilateral panels. Instead of vortex-ring elements, we now place on each panel a source with a constant strength $\sigma$. The velocity potential of this source on an arbitrary point in space $\boldsymbol{P}$ is then obtained as

$$
\Phi(\boldsymbol{P})=\frac{\sigma}{4 \pi} \int_{S} \frac{d S}{\sqrt{\left(\epsilon-\epsilon_{0}\right)^{2}+\left(\eta-\eta_{0}\right)^{2}+\xi^{2}}} .
$$

The coordinates in the local panel coordinate system are given by $\eta, \epsilon$, and $\xi$, whereby $\eta$ and $\epsilon$ are in the panel plane and $\xi$ is the vertical coordinate. The index 0 denotes the collocation point position on the panel. The area of the panel is denoted by $S$.

The solution to this integral has been derived by Hess and Smith [? ] for three-dimensional, closed bodies and the final equations have been documented by Katz and Plotkin [? , Chapter 10.4]. The induced velocities are obtained from the gradient of the velocity potential $\boldsymbol{u}=\partial \Phi / \partial \boldsymbol{P}$. 
Similar to the UVLM, we now enforce the non-penetrating boundary on all collocation points, leading to the linear set of equation $\mathcal{A}_{n l} \sigma_{n l}=-\boldsymbol{B}_{n l}$ with the subindex $n l$ for non-lifting bodies. For this equation, a unique solution for the source strengths exists. From the resulting source strength, the induced velocities on each collocation point can be computed with

$$
\boldsymbol{U}_{i}=\Sigma_{j=1}^{N_{j}} \boldsymbol{u}_{i j} \sigma_{j}+\boldsymbol{U}_{\infty, i}
$$

where $\boldsymbol{u}_{i j}$ is the velocity induced by the source on panel $j$ with the source strength $\sigma_{j}$ on the collocation point $i$ and $N_{j}$ is the total number of panels.

Finally, the resulting forces on each panel can be computed by $\boldsymbol{F}=-\frac{1}{2} \rho_{\infty} C_{p} S U_{\infty}^{2} \cdot \boldsymbol{n}$ with the pressure coefficient

$$
C_{p}=1-\Sigma_{j=1}^{N} \frac{\left|\boldsymbol{U}_{i}\right|^{2}}{\left|\boldsymbol{U}_{\infty i}\right|^{2}}
$$

\section{Steady Coupling}

Next, we couple the aerodynamics for the lifting surfaces and non-lifting bodies. Therefore, we exploit that both underlying models are based on potential flow theory, satisfying the Laplace equation. The Laplace equation is a linear function enabling a superposition of both potential functions. This superposition results in the linear set of equations

$$
\left[\begin{array}{cc}
\mathcal{A}_{l} & \mathcal{A}_{n l, l} \\
\mathcal{A}_{l, n l} & \mathcal{A}_{n l}
\end{array}\right]\left[\begin{array}{c}
\boldsymbol{\Gamma} \\
\sigma
\end{array}\right]=-\left[\begin{array}{c}
\boldsymbol{B}_{\boldsymbol{l}} \\
\boldsymbol{B}_{\boldsymbol{n} l}
\end{array}\right],
$$

where $\mathcal{A}_{l}$ represents the AIC of the vortices on the lifting surface panels and $\mathcal{A}_{n l}$ the AIC of the sources on the non-lifting surface panels. The other two matrices contain the influence of one surface type on the other. More precisely, $\mathcal{A}_{l, n l}$ contains the AIC of the vortices on the non-lifting surface panels, and $\mathcal{A}_{n l, l}$ the AIC of the source panels on the lifting surface panels. The same coupling approach is used to consider interactions between different lifting surfaces like wing-tail configurations.

The wing-fuselage junction requires special treatment. The reason being the abruptly terminated lifting surface at the junction, which leads to a discontinuity in a vortex distribution discretized with vortex rings. This discontinuity would cause non-physical vortex shedding at the wing root [? ]. Consequently, the lifting surface panels, including the wake panels, are further extended inside the fuselage to the symmetry plane, where the non-zero bound vorticity strength are counteracted by its symmetric component. We call the lifting surface panels inside the fuselage phantom panels on which the non-penetrating boundary condition, in contrast to bounded lifting and non-lifting panels, is not enforced. As a result, we get a geometrical setup as sketched in figure 1

Equation 8 is now extended by these phantom panels, denoted by the index $p$, to

$$
\left[\begin{array}{ccc}
\mathcal{A}_{l} & \mathcal{A}_{n l, l} & \mathcal{A}_{p, l} \\
\mathcal{A}_{l, n l} & \mathcal{A}_{n l} & \mathcal{A}_{p, n l}
\end{array}\right]\left[\begin{array}{c}
\boldsymbol{\Gamma} \\
\boldsymbol{\sigma} \\
\boldsymbol{\Gamma}_{p}
\end{array}\right]=-\left[\begin{array}{c}
\boldsymbol{B}_{l} \\
\boldsymbol{B}_{n l}
\end{array}\right]
$$

Further boundary conditions for the circulation strength on the phantom panels, $\boldsymbol{\Gamma}_{p}$, are needed to obtain a unique solution for this problem. In the present work, $\boldsymbol{\Gamma}_{p}$ is set by linear interpolation between the circulation strengths on the wing-junction panels on each side of the fuselage. Linear interpolation is also used when generating the phantom surface geometry $\zeta_{p}$. More specifically, the panels on each wing junction are connected with a straight line, meaning that sweep and dihedral are not continued. The spanwise discretisation is kept similar in size to that at the junction.

For the coupled model, the force calculation has to be adapted. Both, the equations for steady forces on lifting and non-lifting surfaces (equations 3 and 7) depend on the local induced velocity. This induced velocity must now account for the effect of both vertices and sources.

\section{Sectional Corrections}

The UVLM offers many advantages for the typical aeroelastic applications we consider, where thin lifting surfaces with attached flow prevail, which permits, with reasonable accuracy, to neglect viscous effects. Several modifications have been proposed in the literature to introduce viscous effects [? ? ? ] but the associated high computational cost reduces the effectiveness of the method. Other alternative aeroelastic codes use focus on using 2D strip theory models [? ] 


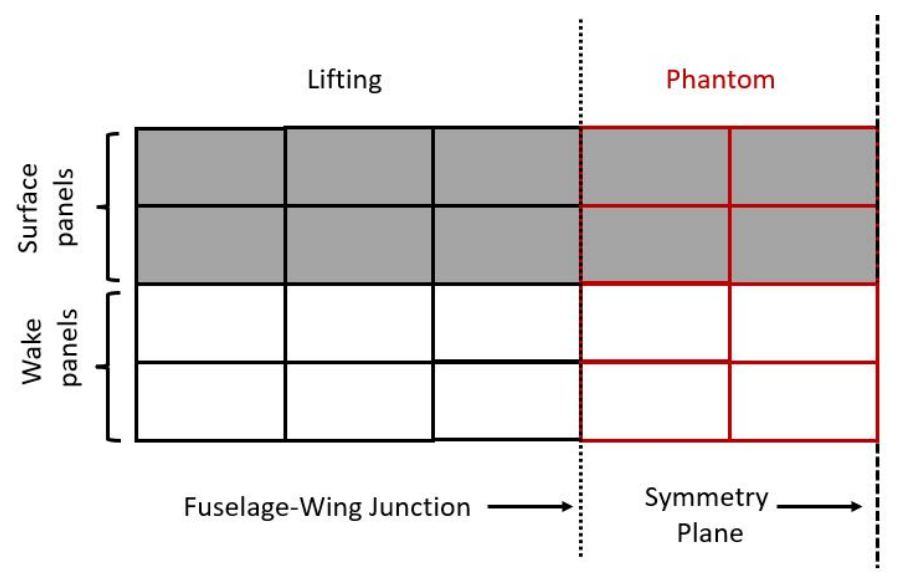

Fig. 1 Resulting lattice geometry for fuselage-wing-junctions using phantom panels.

combined with finite wing corrections to account for 3D effects [? ] or even employ high-fidelity CFD solvers [? ]. In this section, we take an alternative approach where we retain the UVLM to exploit its numerous advantages, such as the panels changing geometry as the wing deforms and the finite wing effects (like induced drag), but we correct the aerodynamic forces within the FSI loop prior to feeding them to the structural subsystem.

The corrections mentioned can account for the following four effects: i) include viscous drag contributions, ii) adjust the lift curve slope for cases where it is far from the potential predicted sectional $C_{l_{\alpha}}=2 \pi$ such as at very low Reynolds numbers, iii) introduce lift deficiency at very high angles of attack near the onset of stall (static effect only) and iv) introduce bespoke lift, drag and pitching moment corrections for sections fitted with control surfaces.

These are implemented within the aeroelastic FSI loop at a sectional level, i.e. for each airfoil section polar data consisting of the two-dimensional lift, drag and pitching moment as a function of the local angle of attack is provided. This method is based on blade element momentum theory philosophy in which forces are computed from look-up tables of steady lift, drag and moment force coefficients, parametrised by the angle of attack.

Although all corrections are based on the effective sectional angle of attack, that angle is not well defined with complex kinematics, thus we turn to the sectional lift coefficient as an appropriate surrogate. First the relative flow velocity at the $i$-th airfoil section is computed, taking into consideration the external flow velocity and the structural motion (both rigid and flexible) as

$$
\boldsymbol{u}_{\mathrm{rel}, i}^{G}=\overline{\boldsymbol{U}}_{\infty, i}^{G}-C^{G A}(\chi)\left(\dot{\boldsymbol{\eta}}_{i}^{A}+\boldsymbol{v}^{A}+\tilde{\boldsymbol{\omega}}^{A} \boldsymbol{\eta}_{i}^{A}\right)
$$

where $\overline{\boldsymbol{U}}_{\infty, i}^{G}$ denotes the average external velocity across all UVLM lattice vertices at the section. The structural motion is defined by the displacement and velocities at the node, $\boldsymbol{\eta}_{i}$ and $\dot{\boldsymbol{\eta}}_{i}$ and the rigid body linear and angular velocity components $\boldsymbol{v}^{A}$ and $\omega^{A}$, respectively, with $(\tilde{\bullet})$ denoting the skew-symmetric matrix. All variables expressed in the aircraft frame $A$ are projected onto the inertial frame $G$ by means of $C^{G A}(\chi)$, which depends on aircraft orientation parametrised by the quaternion $\chi \in \mathbb{R}^{4}$. Thence, we define the transformation from the local material frame $B$ onto the local stability frame $S$ as $C^{S B}\left(\boldsymbol{u}_{\mathrm{rel}, i}^{B}\right)$ to compute the drag, sideforce and lift from the UVLM forces as

$$
\left[\begin{array}{l}
D \\
Y \\
L
\end{array}\right]_{i}=C^{S B} \boldsymbol{F}_{i}^{B},
$$

where $\boldsymbol{F}_{i}^{B}$ are the sectional aerodynamic forces from the UVLM mapped onto the local frame $B$. Finally, the sectional lift coefficient, our surrogate to the effective angle of attack, is found simply as

$$
C_{l, i}=\frac{L}{0.5 \rho\left|\boldsymbol{u}_{\mathrm{rel}, i}^{G}\right|^{2}\left|\boldsymbol{c}_{i}^{B}\right|\left|\boldsymbol{b}_{i}^{B}\right|}
$$

with $\rho$ as the air density and $\boldsymbol{b}_{i}$ being a sectional span vector equal to the spanwise distance between adjacent structural nodes. Knowing the sectional lift coefficient permits us to calculate the effective angle of attack the local airfoil is 
experiencing, i.e. including the downwash effect, using

$$
\alpha_{\text {effective }}=\frac{C_{l, i}}{2 \pi}+\alpha_{0, \text { camber }}
$$

where $\alpha_{0, \text { camber }}$ is the potential flow theory prediction of zero-lift angle of attack for thin, cambered airfoils [? , p. 69]. Equation (13) is computing the ratio between the 3D lift from the UVLM and what otherwise would have been obtained on an infinite wing, which in the case of potential flow methods is equivalent to a $2 \pi$ lift curve slope. This method provides a suitable alternative in computing the effective angle of attack at each section which accounts for finite wing effects. The lift is now trivial to correct, be it for the case of very low Reynolds flows or because of the high angle of attack. The entire scalar from the UVLM is replaced by that extracted from the polar data, since the 3D effect is included through the effective angle of attack

$$
L=0.5 \rho\left|\boldsymbol{u}_{\mathrm{rel}, i}^{G}\right|^{2}\left|\boldsymbol{c}_{i}^{B}\right|\left|\boldsymbol{b}_{i}^{B}\right| C_{l, \text { polar }}\left(\alpha_{\text {effective }}\right)
$$

The viscous drag contribution is extracted from the airfoil polar $C_{d}$ vs $C_{l}$ curve, by interpolating the local lift coefficient to find the local viscous drag and include it to the UVLM (induced) drag

$$
D=D_{\mathrm{UVLM}}+0.5 \rho\left|\boldsymbol{u}_{\mathrm{rel}, i}^{G}\right|^{2}\left|\boldsymbol{c}_{i}^{B}\right|\left|\boldsymbol{b}_{i}^{B}\right| C_{d, \text { polar }},
$$

and, as opposed to the lift correction, the drag from the UVLM which is the lift induced drag is retained and only the viscous contribution from the polar added.

Finally, the corrected pitching moment is computed in a similar manner to the lift, adding the pure moment due to viscous effects and moment due to viscous drag to the circulatory moment as

$$
M=M_{\mathrm{UVLM}}+M_{\text {polar }}\left(\alpha_{\text {effective }}\right)+\tilde{\boldsymbol{r}}^{S} D_{\text {polar }} \boldsymbol{x}^{S}
$$

Alternatively, if choosing to also correct the lift as in (14), the pitching moment from the UVLM, $M_{\text {UVLM }}$ in (16), can be replaced by $\tilde{\boldsymbol{r}}^{S} L_{\mathrm{polar}} z^{S}$, where $\boldsymbol{r}^{S}$ is the moment arm between the nodal position $\eta_{i}$ and the quarter chord point.

Control surface effects can be introduced, in the same manner, following a principle of superposition whereby to the forces computed above a contribution from the control surface given by a polar curve as a function of its deflection is added to the lift, drag and pitching moment.

The corrections of the aerodynamic forces are effectively performed as a post-process to the UVLM, therefore, the circulation field of the UVLM is not updated after the forces are corrected. This could be performed iteratively to have a UVLM circulation field that matches the corrected forces to have a more accurate three-dimensional velocity field. However, this is considered a second order effect and our scheme deemed appropriate as a first approximation to introducing viscous effects from polar data.

The updated forces in the local stability frame can now be projected back onto the local material frame $B$ using the inverse of (11) and fed to the structural subsystem. These corrections introduce a simple procedure of minimal computational cost where the benefits of the UVLM are merged with polar data to add the viscous effects which are otherwise cumbersome to include. that Section III.B will showcase this as an example on simple wings.

\section{Wake Convection Algorithm}

The wake $\partial \Omega_{w}$ is the surface containing the vorticity that has been generated by solid surfaces $\partial \Omega_{s}$ and shed at the trailing edge (Fig. 2). In a region $\Sigma$ (with boundaries $\partial \Sigma$ ) of the wake, the circulation $\Gamma$ is a measure of the vorticity according to Stokes' theorem

$$
\Gamma=\oint_{\partial \Sigma} u \cdot d l=\iint_{\Sigma} \nabla \times u \cdot d \sigma
$$

Any point $\boldsymbol{x} \in \partial \Omega_{w}$ convects with the flow according to

$$
\boldsymbol{x}(t)=\boldsymbol{x}_{0}+\int_{0}^{t} \boldsymbol{u}(\boldsymbol{x}(s), s) d s
$$

where $\boldsymbol{x}_{0}$ is the initial position and $\boldsymbol{u}$ is the flow velocity that includes the upstream undisturbed $\boldsymbol{U}_{\infty}$ and the vortex-induced velocities. This leads to an accurate computation of the wake, the axial induction and the aerodynamic forces and is 


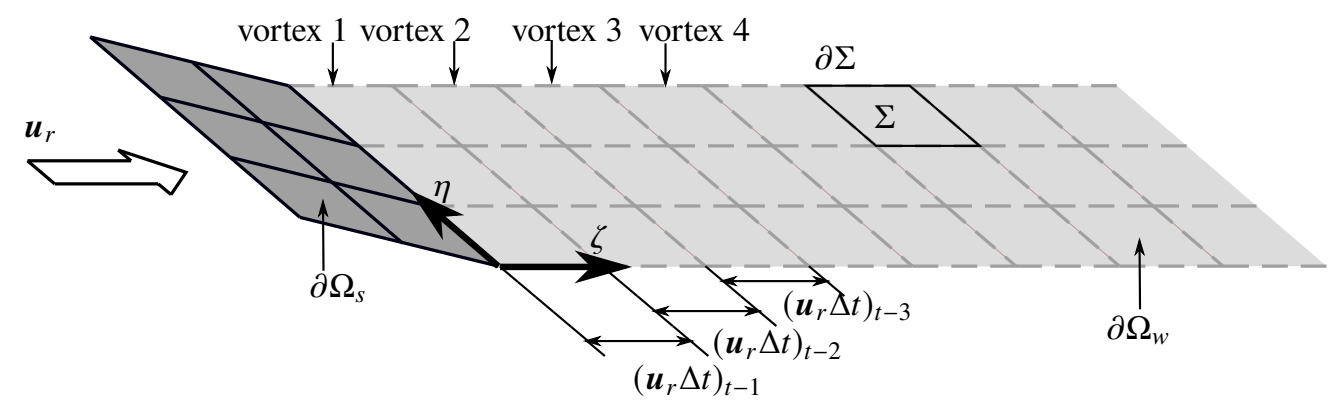

Fig. 2 Solid and wake surfaces discretisation in UVLM

named free wake model. However, the flow velocity can be approximated by the undisturbed velocity for efficiency reasons and is referred to as prescribed wake.

Kelvin's theorem applies to regions of ideal forces free form external forces like the wake. It states that the flow circulation around a closed curve $\partial \Sigma$ does not change with time. Thus, in a Lagrangian description the time evolution of the wake shape and vorticity consist on displacing the closed curves $\partial \Sigma$ according to Equation (18) which retain their initial circulation.

Frames of reference moving with solid surfaces are the standard in aerodynamics in which a convection of the wake circulation $\Gamma$ away from the solid is observed. In this case, an Arbitrary Lagrangian-Eulerian description is more convenient: the wake surface is described by two coordinates $\eta$ and $\zeta$ along the spanwise and streamwise directions, respectively (Fig. 22. Therefore, convection of circulation only occurs along the $\zeta$ coordinate and independently for each streakline $\eta$ according to:

$$
\frac{\partial \Gamma}{\partial t}+u_{r} \frac{\partial \Gamma}{\partial \zeta}=0
$$

where $\Gamma(\zeta, \eta, t)$ is the flow circulation, the module of the relative flow-solid velocity is computed as $u_{r}(\zeta, \eta, t)=$ $\boldsymbol{u}(\zeta, \eta, t) \cdot \hat{\zeta}(\zeta, \eta, t)-\boldsymbol{u}_{s}(0, \eta, t) \cdot \hat{\zeta}(0, \eta, t)$ where $\boldsymbol{u}$ and $\boldsymbol{u}_{s}$ are the flow and known solid trailing edge velocities, respectively and $\hat{\zeta}$, the unit vector in the $\zeta$ direction. This equation closes the vortex problem. However, they are usually solved in a staggered manner such that the latter provides the initial $\Gamma(\eta, \zeta, 0)$ and boundary $\Gamma(0, \zeta, t)$ conditions for the former.

At the beginning of the simulation, the spanwise $\eta$ discretisation is chosen, however, the discretisation in the streamwise direction $\zeta$ is automatically generated at each time step. At each time step (Fig. 3p), solid surfaces move with their velocity $\boldsymbol{u}_{s}$ and wakes are convected by the local flow velocity $\boldsymbol{u}$ generating a gap between the trailing edge and the first wake node. In this gap a new segment equal to $\left(\boldsymbol{u}-\boldsymbol{u}_{s}\right) \Delta t$ is created. By construction, it is parallel to the streamwise direction $\zeta$ and the relative solid-fluid velocity $\left(\boldsymbol{u}_{r}=\boldsymbol{u}-\boldsymbol{u}_{s}\right)$.

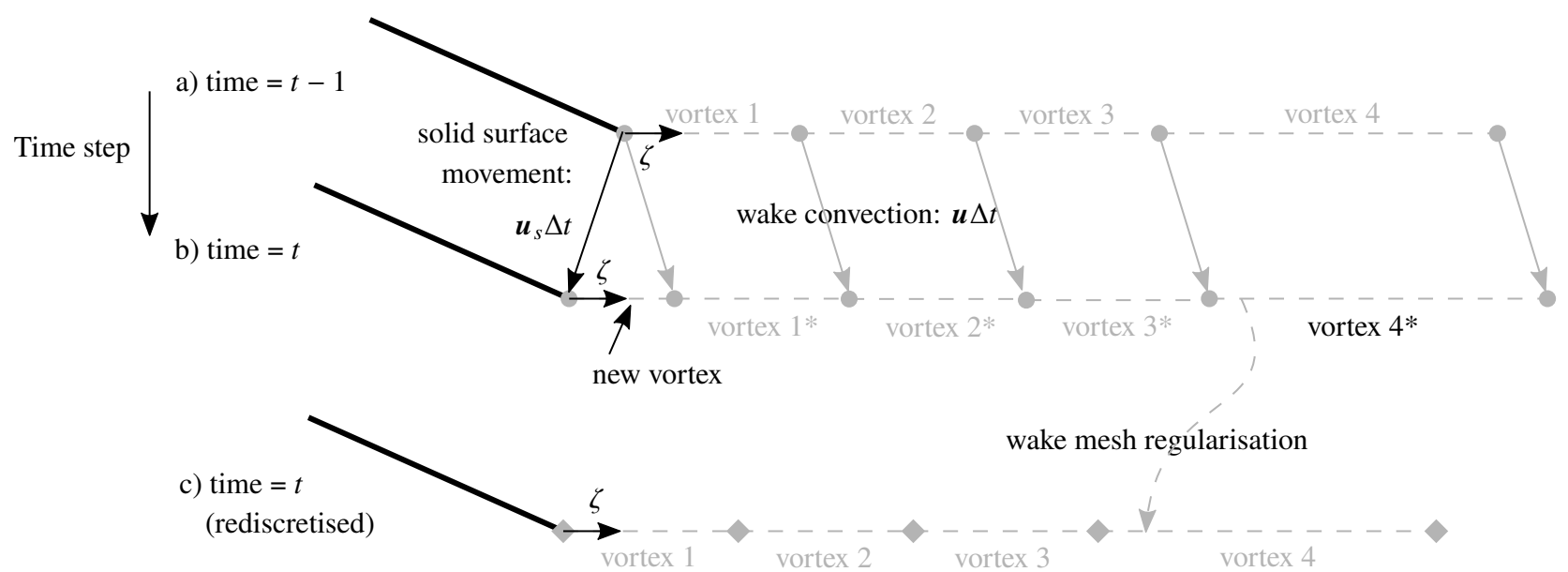

Fig. 3 New non-uniform wake rediscretisation method 
The first order upwind discretisation of $[19]$ is

$$
\frac{\Gamma_{\zeta}^{t+1}-\Gamma_{\zeta}^{t}}{\Delta t}+u_{r} \frac{\Gamma_{\zeta}^{t}-\Gamma_{\zeta-1}^{t}}{\Delta \zeta}=0
$$

with $\Delta \zeta$ the vortex size in the $\zeta$ direction. Rearranging this equation and simplifying by defining $C=u_{r} \Delta t / \Delta \zeta$ :

$$
\Gamma_{\zeta}^{t+1}=\left(1-\frac{u_{r} \Delta t}{\Delta \zeta}\right) \Gamma_{\zeta}^{t}+\frac{u_{r} \Delta t}{\Delta \zeta} \Gamma_{\zeta-1}^{t}=(1-C) \cdot \Gamma_{\zeta}^{t}+C \cdot \Gamma_{\zeta-1}^{t} .
$$

In traditional panel methods [?], $C=1$ is forced by generating the streamwise discretisation as $\Delta \zeta=u_{r} \Delta t$. This simplifies the wake convection equation to a movement of values from one discretisation element to the following:

$$
\Gamma_{\zeta}^{t+1}=\Gamma_{\zeta-1}^{t}
$$

In [? ], we proposed instead a discretisation of Equation (21) that allows the coarser vortices in regions where vorticity has less influence on the forces on solid surfaces (usually far away from them) for which $\Delta \zeta \neq u_{r} \Delta t$ is needed. In free wake models, the computational time grows as $O\left(N_{w}^{2}\right)$ with $N_{w}$ the number of vortices that discretise the wake geometry, thus, the use of coarser vortices will improve computational efficiency.

\section{Verification Studies}

After the detailed theoretical presentation of the non-lifting body model, the polar corrections and the improved discretisation scheme for wake flows in the previous chapter, we now turn to the application of these methods. For first verification proofs, we apply them here to simple demonstration examples.

\section{A. Non-Lifting Body Effects}

The previously described mathematical formulation for the non-lifting body and its coupling with lifting surfaces has been implemented in SHARPy. The results from SHARPy are compared here to experimental and analytical results to verify the implementation and to validate the modeling assumptions. Subsequently, the implementation is applied on a simple wing-fuselage configuration to demonstrate the fuselage effect on the wing's aeroelasticity.

\section{Validation}

First, the non-lifting methodology using constant quadrilateral sources on the wetted surface is validated. An ellipsoid with a thickness ratio of eight is chosen as a first reference case. The body is discretized into 39 spanwise and twelve radial panels. Figure 4 shows the pressure distribution over the spanwise coordinate $x$ divided by the body length $L$. The analytical solution of the pressure distribution over an ellipsoid in potential flow theory is denoted by the solid line and the crosses represent the SHARPy result.

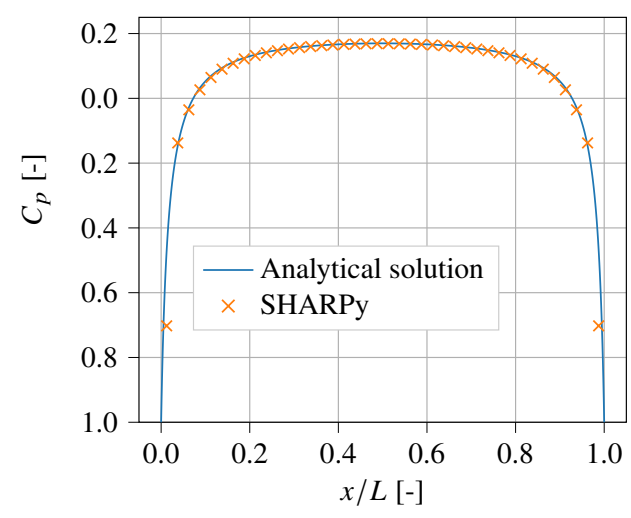

Fig. 4 Pressure distribution over an ellipsoid. 
The figure shows a good agreement of the analytical solutions with the results of SHARPy. Only near the nose and the tail the results deviate slightly, which is caused by the low resolution of the strong local radius change. Overall, however, it proves the correctness of the implementation and the high accuracy even with a small grid resolution.

Second, a mid-wing and low-wing aircraft configuration with a cylindrical fuselage shape serve as additional reference cases. These cases are used to validate the coupling between lifting and non-lifting surfaces and the wing-fuselage-junction handling. The geometrical parameters are defined in figure 5(a) For both configurations, experimental data for the lift distribution has been measured by Körner [? ? ] in incompressible wind tunnel experiments. In Figs. 5(b) and 5(c) the experimental data, marked in blue, is compared to steady aerodynamic results computed with SHARPy with and without a fuselage, marked in green and orange.

For the mid-wing configuration, one sees a slight decrease in the lift coefficient from the wingtip due to the presence of the fuselage. Also close to the junction, a lift drop occurs. In comparison to the experimental data, SHARPy results show a good agreement. Only near the wing-fuselage junction, the sudden drop in lift created by the boundary layer of the fuselage in the experiments is not captured by SHARPy. However, the applied loads near the junction do not significantly affect the structural deformations of the wing. It should also be noted that typical aircraft configurations have an adjusted wing geometry at the junction to reduce drag (e.g. strakes). These detailed geometry adjustments are difficult to represent with a thin-wing assumption for the aerodynamic solver and are not the focus of our analysis method.

For the low-wing configuration, good agreement of the results from the experiments and SHARPy is also observed. Only the numerically obtained lift coefficient values near the junction deviate slightly from the experimental results. In contrast to the mid-wing configuration, a lift increase due to the blockage effect of the fuselage occurs between the junction and $80 \%$ of the wingspan if compared to a wing-only configuration occurs for the low-wing configuration.

The agreement between numerical and experimental results is very accurate. Small deviations due to the airfoil thickness, viscous, and wind tunnel interference effects would be expected as the UVLM cannot capture these effects but the corrections outlined before where not deemed necessary for this problem.

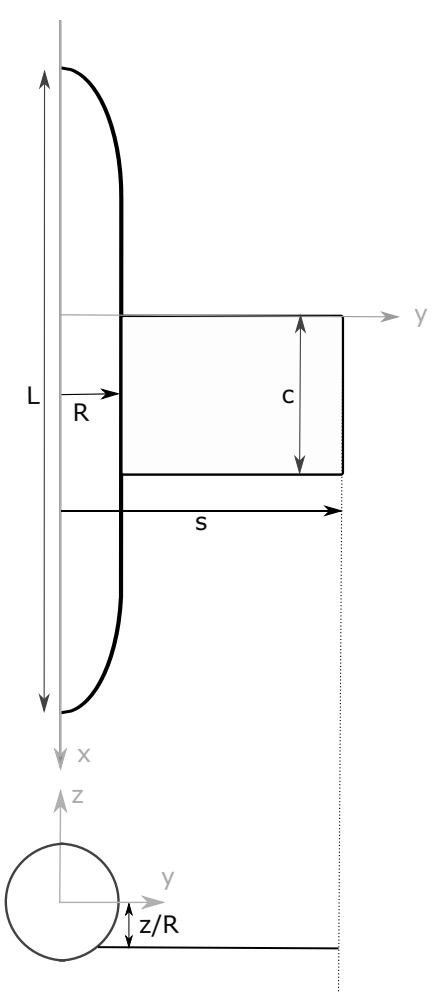

(a) Geometry.

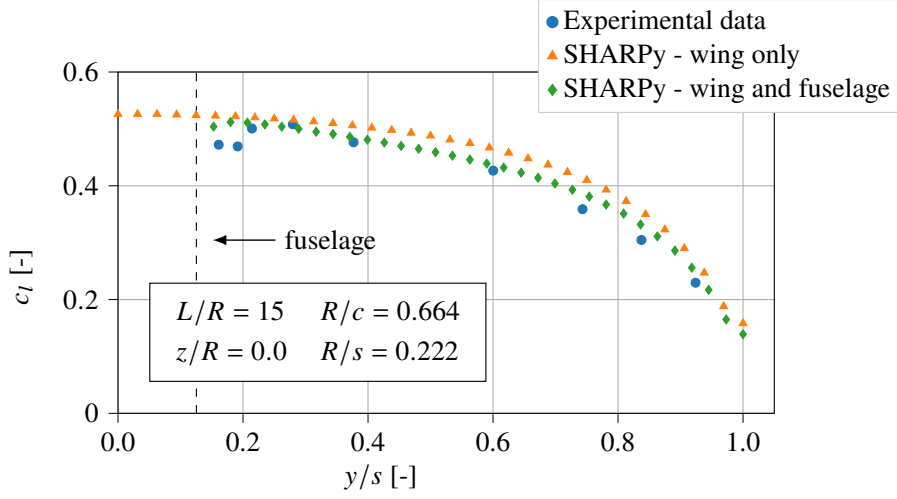

(b) Mid-wing configuration $\left(\alpha=6^{\circ}\right)$.

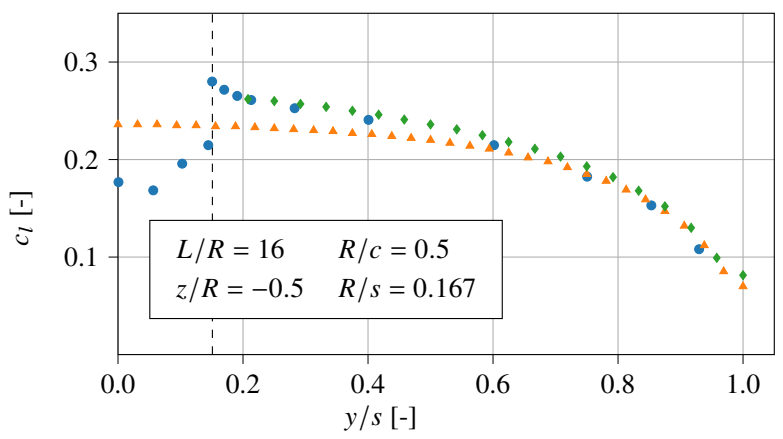

(c) Low-wing configuration $\left(\alpha=2.9^{\circ}\right)$.

Fig. 5 Comparison of lift distribution from experiments [? ? ] and SHARPy. 
Table 1 Structural properties of the mid-wing model.

\begin{tabular}{lccccc}
\hline Component & $E A[\mathrm{Nm}]$ & $G J\left[\mathrm{Nm}^{2}\right]$ & $G A\left[\mathrm{Nm}^{2}\right]$ & $E I_{y}\left[\mathrm{Nm}^{2}\right]$ & $E I_{z}\left[\mathrm{Nm}^{2}\right]$ \\
\hline Wing & $1.0 e 7$ & $1.0 e 4$ & $1.0 e 5$ & $2.0 e 4$ & $4.0 e 6$ \\
Fuselage & $1.0 e 8$ & $1.0 e 5$ & $1.0 e 6$ & $2.0 e 5$ & $4.0 e 7$ \\
\hline
\end{tabular}

\section{Fuselage-Effect on Aeroelasticity}

Now the effect of the fuselage on the aeroelasticity of the mid-wing configuration with a wingspan of $b=2 \mathrm{~s}=5.976 \mathrm{~m}$ and a dynamic freestream pressure of $q_{\infty}=61.25 \mathrm{~Pa}$ is evaluated. For this purpose, coupled, steady simulations are performed using SHARPy for a wing-only and wing-fuselage configuration. For both configurations, the wing structure goes to the symmetry plane. The structural stiffness properties for the wing and fuselage are summarized in table 1 The wing's stiffness constants are multiplied by a factor $\lambda$ to address the effect of the wing's flexibility. The lower $\lambda$ is, the higher is the wing's flexibility.

For the different material stiffnesses, the wing deformation is shown in the $y-z$ plane in figure 6 for the mid-wing configuration with and without a fuselage. For both configurations, the wing deformation is, as expected, larger for smaller $\sigma$ as the material becomes more flexible. If we compare the wing deformation with and without the presence of the fuselage, we can observe that the fuselage barely affects the wing deformation for stiff wings. However, the more flexible the wing becomes, the higher is the deformation with a fuselage than for a wing-only configuration. This is caused by the blockage effect of the fuselage. The blockage is enhanced for larger wing deformations and accelerates the flow over the wing's suction side. As a result, the fuselage causes a lift increment as the wing deformation increases. This lift increase due to the blocking effect has a greater influence on the wing deformation than the extra lift generated at the additional lifting surfaces on the root section of the wing-only configuration. Overall, this result emphasizes the importance of including fuselage effects on a very flexible aircraft.

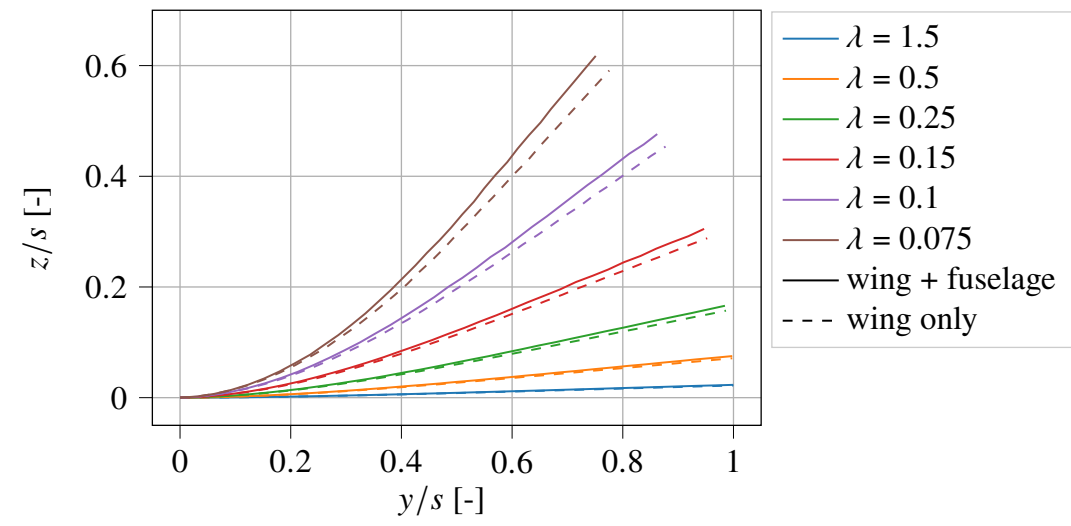

Fig. 6 Vertical displacement of the wing structure for the wing-only and mid-wing configuration.

\section{B. Sectional Corrections}

We evaluate the performance of the sectional corrections described in Sec. II.C. using polar data for a NACA0018 airfoil at a low Reynolds number $R e=50000$ and zero Mach number that has been numerically derived from XFOIL [? ]. XFOIL introduces viscous effects which, for this airfoil, are clearly visible not only in the drag polar but also in the lift curve, where near 0 degrees a substantial increase in gradient is observed (see Fig. 77).

Initially, we validate the approach using an infinite wing in order to converge to $2 \mathrm{D}$ results using the UVLM and compare against the polar data. The simulation is set up in SHARPy using a flat wing of aspect ratio $10^{7}$, with 4 spanwise panels, 8 chordwise panels and a horseshoe wake. The expected UVLM result is that predicted by potential flow thin airfoil theory for a flat plate $\left(C_{L}=2 \pi \alpha, C_{D}=0\right.$ and $\left.\left.C_{M}\right|_{0.25 c}=0\right)$. This is shown in Fig. 7 The corrected UVLM follows exactly the source polar data, and is effectively validating (13) since the local lift coefficient is equal to 

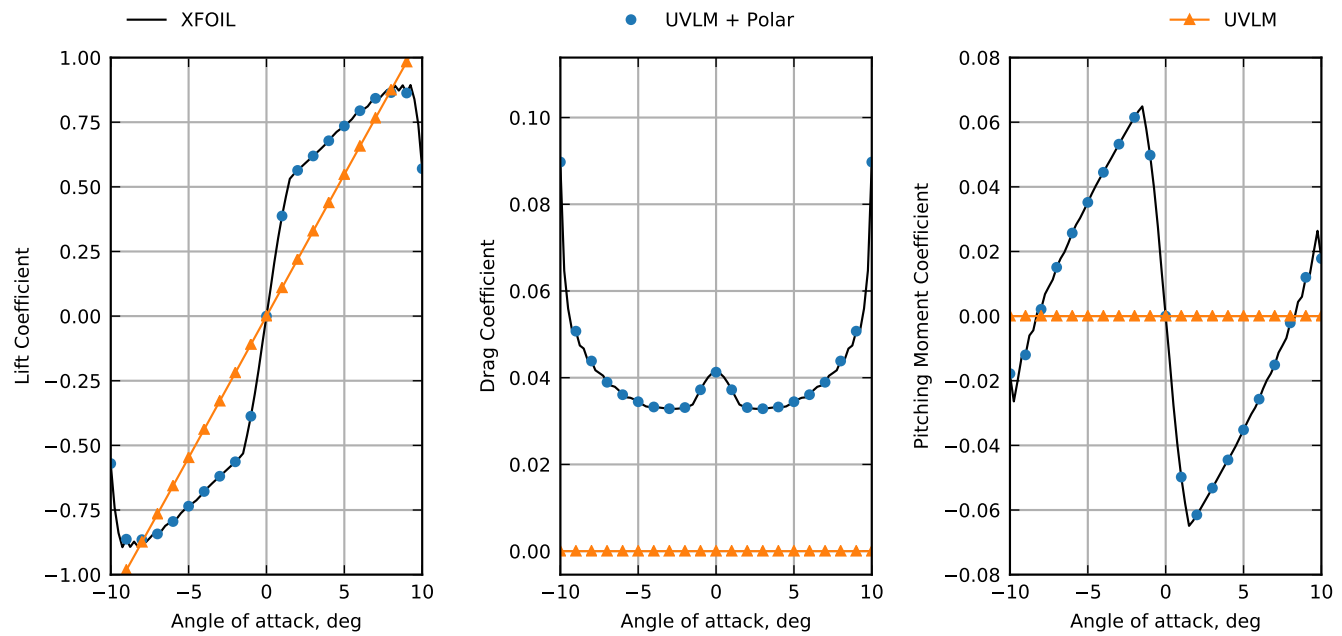

Fig. 7 Lift, drag and moment curves for an infinite wing comparing, in SHARPy, the polar data, the UVLM with polar sectional corrections, and the standalone UVLM.

the 2D sectional lift. This yields an effective angle of attack without computing it explicitly but simply using the lift coefficient as surrogate.

Turning now to finite wings, a wing of aspect ratio 8 has been chosen. The discretisation is again 8 chordwise panels, 4 spanwise panels and a horseshoe wake. This three-dimensional case now introduces finite wing effects, which in the UVLM translates to induced drag and a reduction in lift, with the results shown in Fig. 8. It can be seen that the UVLM lift curve slope is about $80 \%$ of the infinite wing case (in line with the approximation from Prandlt's lifting line theory) and that the drag curve is now parabolic, accounting for the induced drag effect.

The polar corrected UVLM on the finite wing is now being affected too by the 3D effects. Note the 2D data is shown for reference to illustrate the effect. The lift curve slope reduction is again $80 \%$ of the $2 \mathrm{D}$ case at 0 degrees angle of attack, and the drag sees the addition of viscous and induced drag effects. This affects as well the pitching moment, which differs significantly from the inviscid solution.
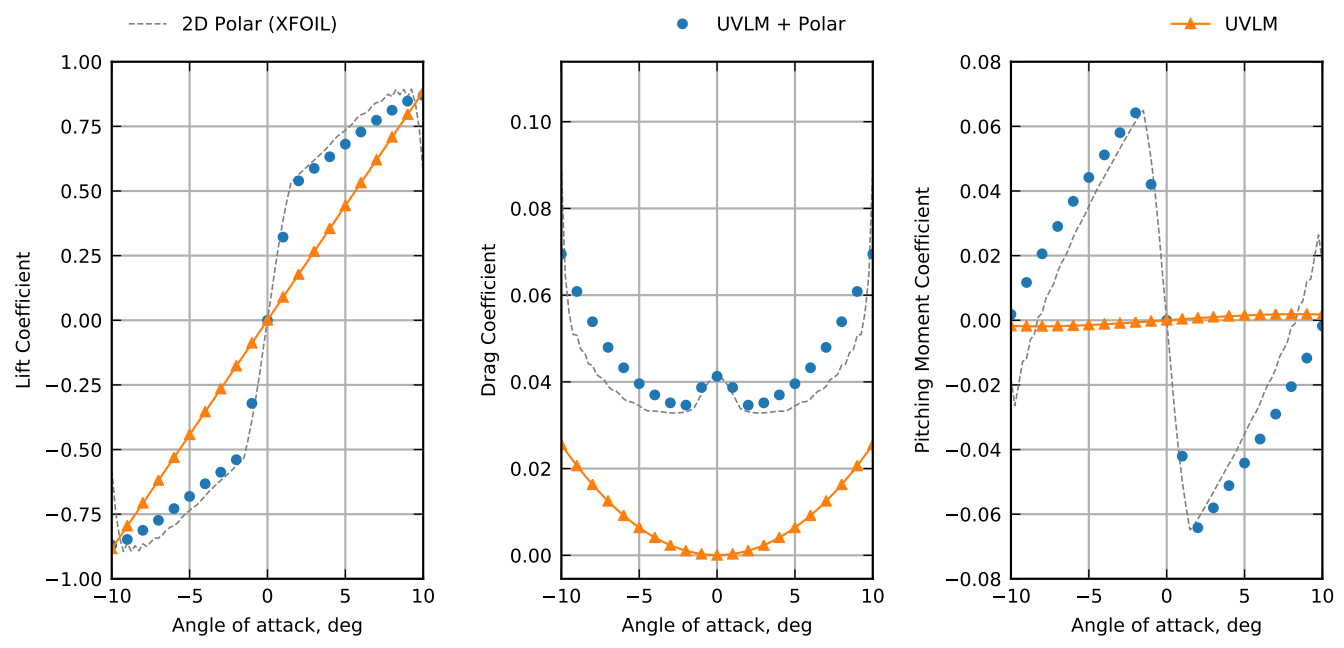

Fig. 8 Lift, drag and moment curves for a aspect ratio 8 wing comparing the UVLM with polar sectional corrections against the standalone UVLM, with the 2D polar data displayed for reference. 
This capacity to introduce polar data and correct the UVLM derived forces efficiently can be used to improve the predictions on the dynamical response of very flexible aircraft (on the phugoid mode, for example) or include wind-tunnel derived data for clamped, very flexible wings, amongst other uses.

\section{Efficient Wake Convection}

For the verification of the new wake discretization scheme, we will us an infinite rigid wing, with chord $c$ and, initially, at zero angle of attack as a simple example, shown in figure 3. Two different wake discretisations have been studied: one traditional $C=1$ approach and another with coarser vortices away from the wing (Figure 9(a)) that significantly reduces the number of vortices required. The airfoil has been exposed to a step gust (Küssner gust) with horizontal relative velocity $u_{r}$. The ratio between instantaneous lift coefficient $c_{L}$ and its value in steady state $c_{L_{\mathrm{ss}}}$ is shown in Fig. 9(b) with respect to the reduced time

$$
\bar{t}=\frac{2 t u_{r}}{c} .
$$

Both models give identical results and match the analytical solution.

At each time step, one vortex is created (as explained above) but the last vortex is removed (vortex $4^{*}$ in Fig. 33) for computational convenience. In the new wake discretisation proposed, the size of the vortices is not uniform (vortex $4^{*}$ is larger than the new vortex), thus, the wake length is reduced at each time step. This shortening should be avoided by performing an additional step of mesh regularisation after the wake convection. This step can be performed independently on each streakline and guarantees that the distance from each vortex corner to the trailing edge (Fig. 3r) is kept equal to the initial one (Fig. 33). This algorithm conserves the total vorticity in the wake and, thus, satisfies Kelvin's law. For straight wakes a simple linear interpolation scheme in Cartesian coordinates is sufficiently accurate. For wind turbine helicoidal wakes more complex algorithms are needed [? ].

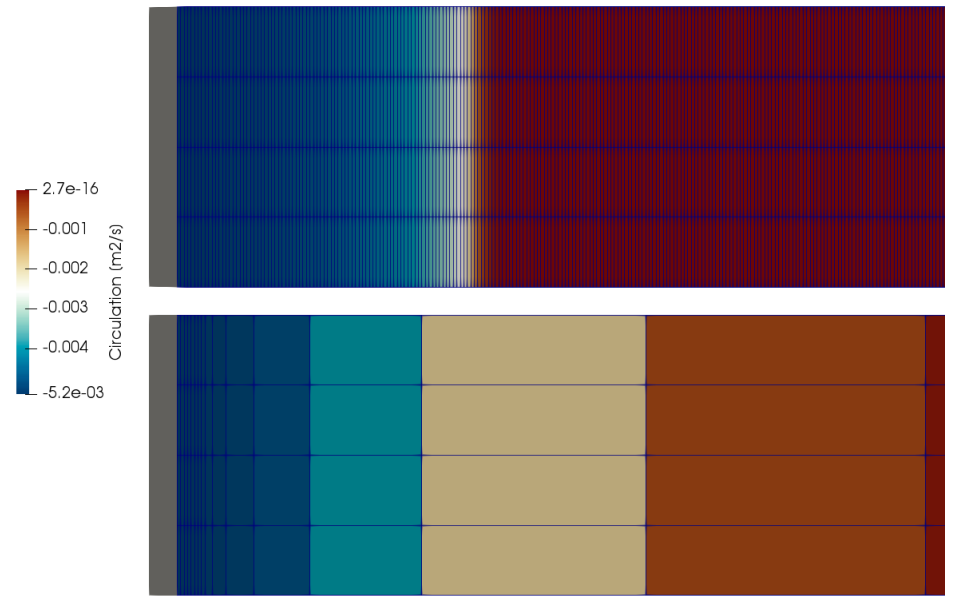

(a) Constant (top) and variable size (bottom) of the wake discretisation

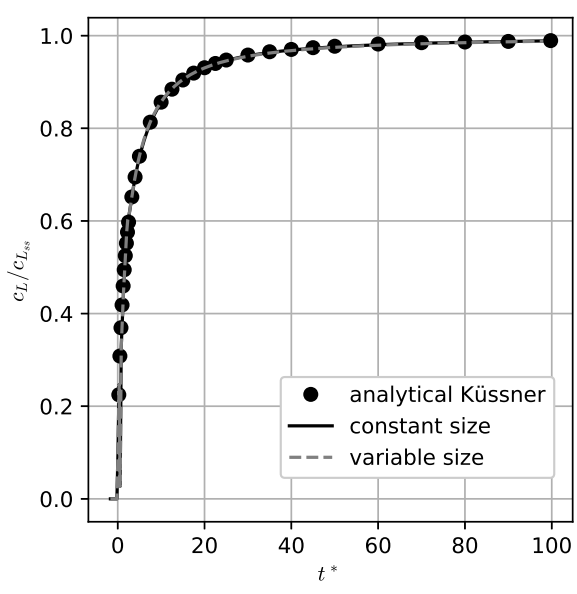

(b) Lift coefficient

Fig. 9 Scheme and lift force in a Küssner gust with traditional and new wake discretisation schemes 


\section{FLEXOP Demonstrator}

Finally, the non-lifting body model and polar corrections are combined and applied to a demonstrator model, more precisely the FLEXOP UAV [? ? ? ? ? ? ]. The FLEXOP aircraft features a tapered and swept wing, a fuselage with varying elliptical cross-sections, and a V-tail and has been modelled in SHARPy as shown in figure 10. This implementation process is first presented in this section along with assumptions made. Second, a verification of the results obtained from SHARPy is carried out with the work of Sodja et al [? ]. Third, the influence of the fuselage on the wings' aeroelasticity and the effect of the presented polar corrections are demonstrated, considering steady effects.

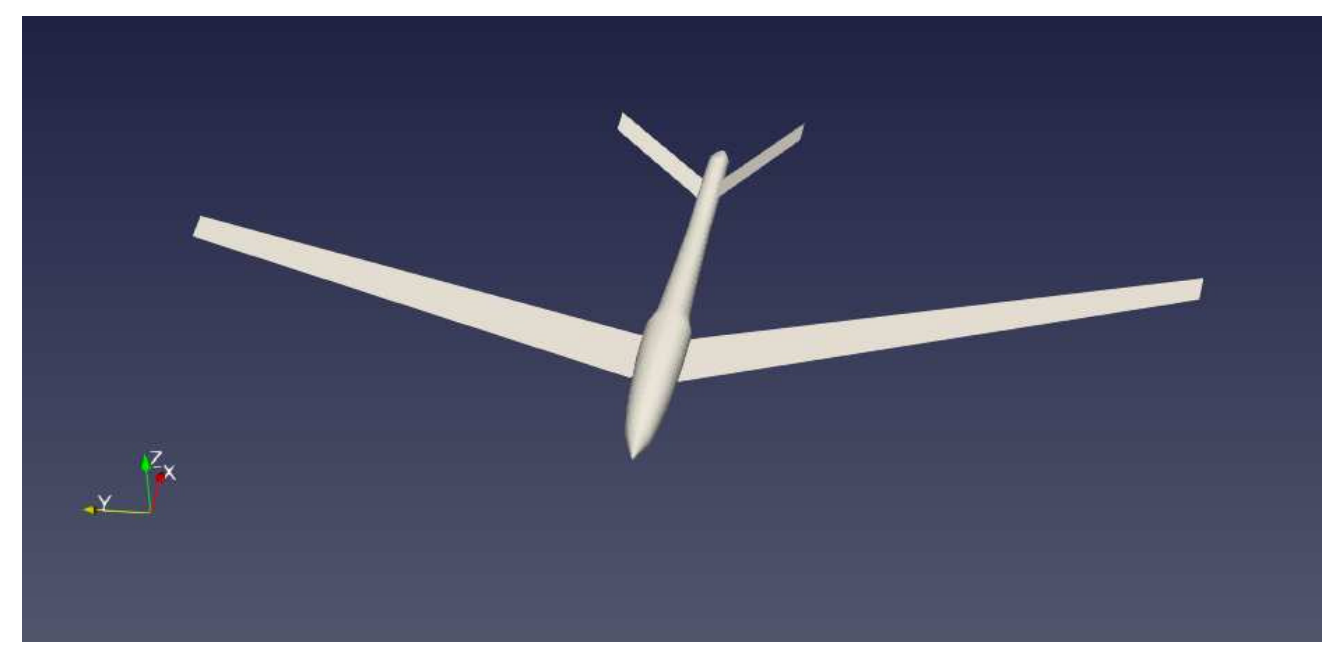

Fig. 10 Three-dimensional visualization of the FLEXOP model in SHARPy.

\section{A. Model Setup}

For the implementation of the FLEXOP model in SHARPy, the work of Sodja et al [? ] serves as a primary reference. The wing model has a sweep angle of $20^{\circ}$, a wingspan of $b=7.07 \mathrm{~m}$, and the wing chord reduces from $0.471 \mathrm{~m}$ at the chord to $0.236 \mathrm{~m}$ at the tip. The stiffness and mass properties of the wing structure of the investigated reference wing have been used as a first instance. Its published jig twist and shear center as well as shared airfoil camber and lumped mass information have been included in the model as well. The airfoil thickness reduces from $10 \%$ at the root to $8 \%$ at the tip and becomes important for the polar corrections applied later. The total mass of the wing is given as $24,1 \mathrm{~kg}$, with $11 \mathrm{~kg}$ being the structural mass.

The fuselage and tail model, in turn, are based on several assumptions since details of both components could not be acquired yet and are, in general, less important than the wing for our work. The geometry of both components, for instance, has been reproduced from CAD sketches found in [? ]. The V-tail has an opening angle of $35^{\circ}$, a sweep angle of $19.5^{\circ}$, a span of $1.318 \mathrm{~m}$, as well as a root chord of $0.217 \mathrm{~m}$ which tapers to $0.180 \mathrm{~m}$ at the tip. The fuselage is assumed to be $3.44 \mathrm{~m}$ long and the offset between wing and nose is $0.884 \mathrm{~m}$ and between nose and tail as $2.86 \mathrm{~m}$.

In addition, no significant flexibility is assumed for the fuselage and tail. Therefore, the stiffness of their structure is considered much higher than that of the wing. Realistic values are assumed for the mass distribution of the two components.

\section{B. Steady Verification and Fuselage Effects}

The purpose of this section is to verify the FLEXOP wing model and to demonstrate the aeroelastic effects of the wing and the influence of the fuselage on these effects. Therefore, steady-state coupled aeroelastic simulations of the FLEXOP wing are performed for a wing-only and a wing-fuselage configuration. The results obtained with SHARPy are compared with those published by Sodja et al [? ]. Their results are computed with an aeroelastic simulation tool that incorporates aeroelastic tailoring and morphing effects and uses UVLM as an aerodynamic solver [? ? ].

The results presented in [?] are obtained for a free-stream velocity of $U_{\infty}=45 \mathrm{~m} / \mathrm{s}$, a flight altitude of $800 \mathrm{~m}$, corresponding to a free-stream density of $\rho_{\infty}=1.1336 \mathrm{~kg} / \mathrm{m}^{3}$, and two different loading conditions are considered, more precisely: cruise flight with a loading factor of $1 g$ and a highly inclined wing with a loading factor of $5 g$. 


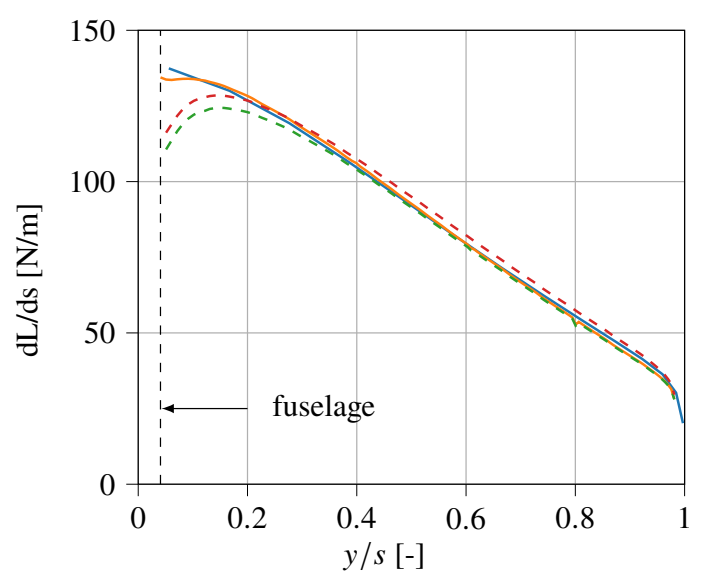

(a) $1 \mathrm{~g}$

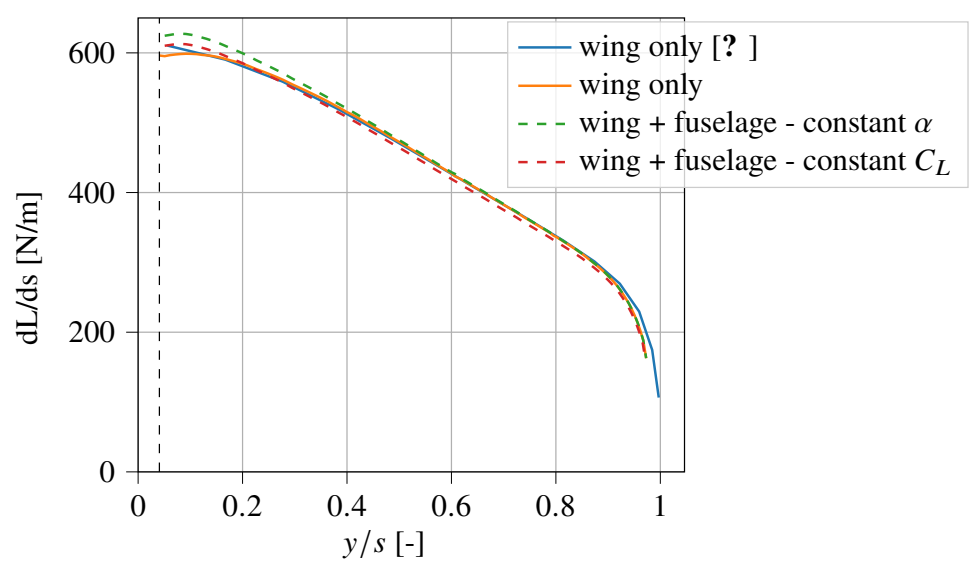

(b) $5 \mathrm{~g}$

Fig. 11 Spanwise lift distribution of the wing for the FLEXOP model with and without considering fuselages.

In a first step, a grid convergence study is performed to ensure modal convergence of the beam and aerodynamic convergence of the lifting surface. As a result, 36 elements per wing in the spanwise direction and 16 vortex surfaces in the chordwise direction are chosen. It should be noted that the chordwise discretization is highly sensitive. The reason being is the discretization of the cambered airfoil, where finer grids capture more camber effects and thus result in more lift.

In the next step, the lift distribution of the wing-only configuration is matched to the lift distribution of the reference case to obtain a comparable structural load response. Then, the wing-fuselage configuration is simulated with the same angle of attack. The obtained lift distributions are summarized in Figure 11 .

For both load factors, the lift distribution in spanwise direction for the wing-only configuration, marked in orange, matches almost perfectly the distribution of the reference case, marked in blue. Only at the transition from swept to unswept wing does a slight deviation in lift occur. For the wing-fuselage configuration at the same $\alpha$, marked in green, different effects on the lift distribution are observed for the different load cases. While for the $1 g$ case the lift decreases near the joint, similar to the mid-wing configuration analyzed before, lift increases in this region for the $5 g$ load case. Similar behavior has been observed in experiments with a mid-wing configuration [? , Chapter 10.213], for which a significant increase in lift near the fuselage junction has occurred in the experiments at similar magnitudes of $\alpha$ as well.

This non-negligible change in lift, especially for the $1 g$ case, means that a change in $\alpha$ is required to maintain the same load factor. The resulting lift distribution for the corrected $\alpha$ is highlighted in red and results in a slight change in lift over the entire wingspan.

The resulting spanwise OOP deformation is shown next in figure 12. For the $1 g$ load case, the OOP deformation of the wing-only configuration deviates minimally from the reference case. For the $5 g$ load case, however, a deviation of almost $4 \%$ of the wingspan occurs in the OOP bending. This deviation might arise due to differences in the structural models used.

Further, we see that the change in lift at the junction caused by the fuselage at the same $\alpha$ does not affect the OOP deformation for any load case. This contradicts the expectations arising from the results of the mid-wing configuration presented in section III.A.2. However, considering the different dimensions of both models, the FLEXOP model has a much narrower fuselage and wider wingspan. Specifically, the ratios between maximum radius and wingspan are for the $\operatorname{FLEXOP}(R / s=8.4 \%)$ almost three times smaller than for the mid-wing configuration $(R / s=22.2 \%)$. Note that for typical commercial aircraft this ratio is about $R / s \approx 18 \%$. The A380, for example, has a ratio of $R / s=17.8 \%$ and the A350 of $R / s=17.6 \%$.

For the corrected $\alpha$, slightly larger OOP deformations occur for the wing-fuselage configuration for the $1 \mathrm{~g}$ load factor. For the $5 \mathrm{~g}$ load factor, no difference becomes apparent because the magnitude of the deformations is here much larger than the magnitude of the change in $\alpha$.

In contrast to the OOP bending, the torsional bending, shown in Figure 13, is different for all configurations. Comparing first the reference and the wing-only configurations, small differences in torsion start at $y / s \approx 0.5$. For the $1 \mathrm{~g}$ load case, this difference grows toward the wingtip, where the magnitude of the torsional deformation is about 


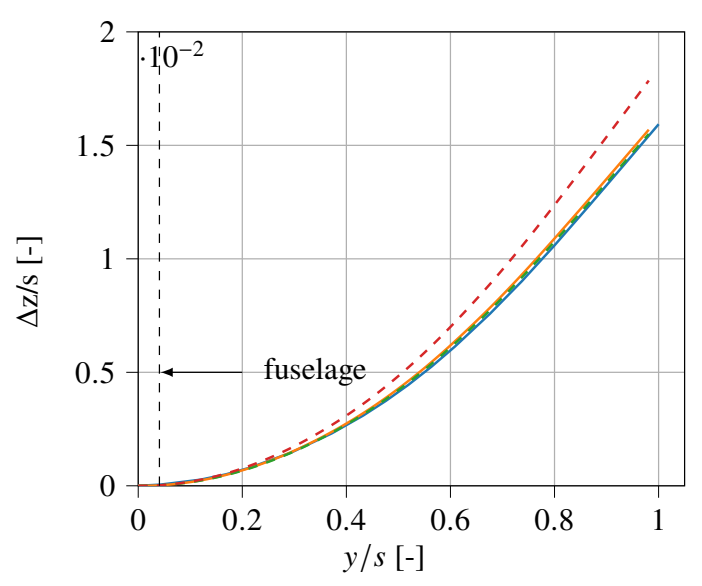

(a) $1 \mathrm{~g}$

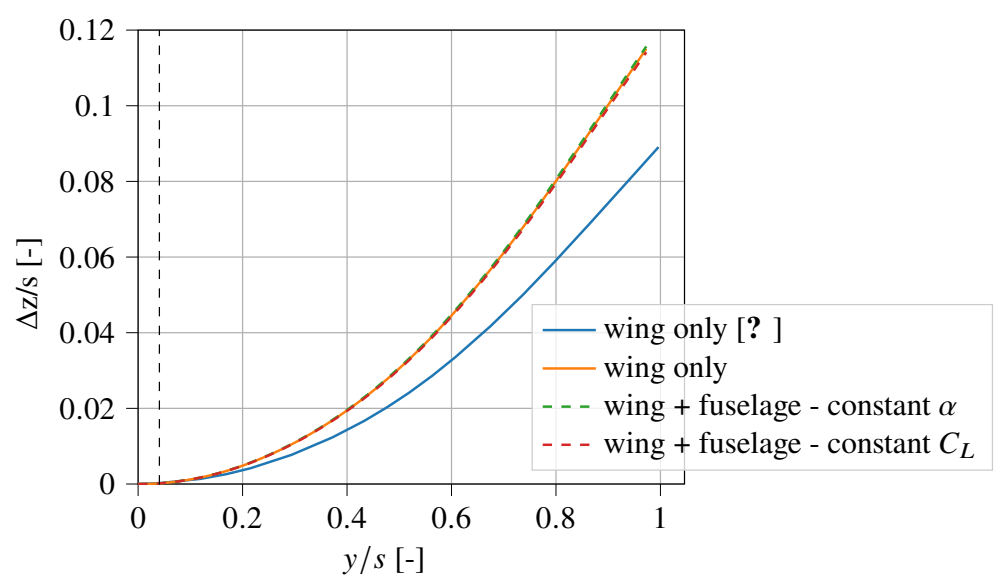

(b) $5 \mathrm{~g}$

Fig. 12 Spanwise OPP deformation of the wing for the FLEXOP model with and without considering fuselages.

$\Delta \Theta=0.065 \mathrm{deg}$ higher than in the reference case. For the $5 \mathrm{~g}$ load case, the difference grows slightly at first until at $y / s=0.8$ the torsional deformation increases linearly for the wing-only configuration, resulting in a difference of $\Delta \Theta=0.077 \mathrm{deg}$. It should be noted that these differences in torsional bending are, however, negligibly small.

Focusing next on the fuselage effect, we see for a present fuselage at $1 g$ the torsional bending is shortly after aerodynamic forces become present higher in magnitude than the wing-only configuration at the same $\alpha$. The difference that arises there remains approximately constant until the wingtip, including a small difference drop at $y / s \approx 0.4$. In the case of a corrected $\alpha$, as for the OOP deformation, the torsional bending increases noticeably in magnitude.

For the load factor $5 g$, the same torsional deformations are obtained with SHARPy's wing-only model as for [? ]. The fuselage effects are here negligible small for both angles of attack as expected for only small deviations in the spanwise lift distribution.

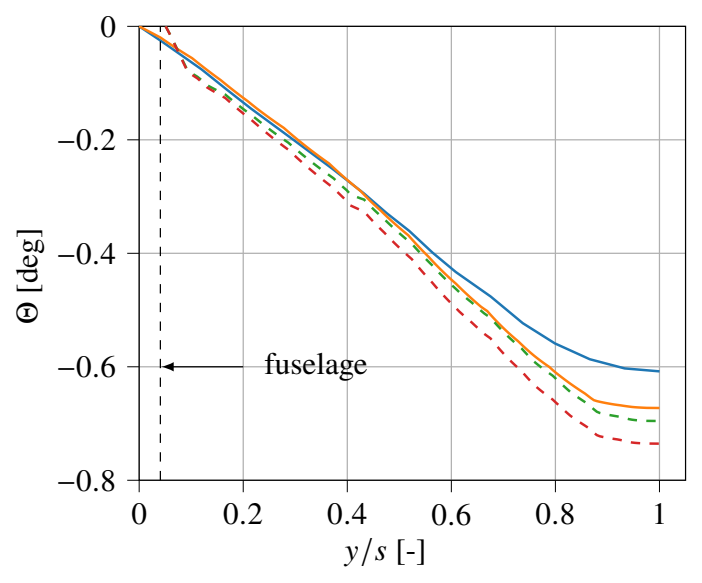

(a) $1 \mathrm{~g}$

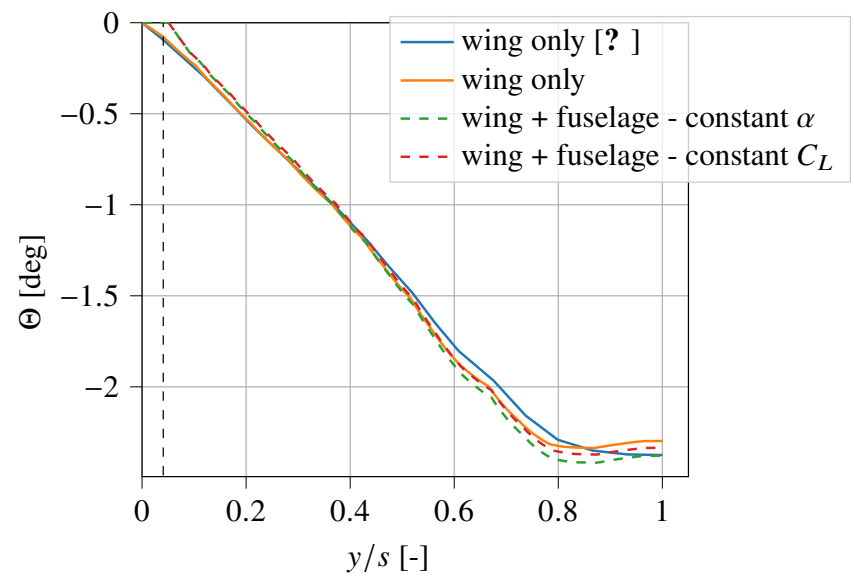

(b) $5 \mathrm{~g}$

Fig. 13 Spanwise torsional deformation of the wing for the FLEXOP model with and without considering fuselages.

\section{Aircraft lift, drag polar and moment with fuselage effects and airfoil data corrections}

The final case study aims to bring together all the enhancements to the UVLM method on the FLEXOP demonstrator. Therefore, we will include non-lifting body effects from the fuselage and airfoil polar corrections to introduce viscous 
drag effects.

We commence discussing the airfoil polar corrections added to the UVLM. The FLEXOP demonstrator wing is based on a asymmetric profile of smoothly varying thickness, from 10\% at the root to $8 \%$ at the tip [? ] which have been analyzed using XFOIL's viscous solver to obtain the sectional lift, drag and moment coefficients in a range of angle of attack. At the cruise condition specified in Sec. IV.B a Reynolds number $\operatorname{Re}=1.3 \times 10^{6}$ and a fully turbulent boundary layer are assumed. The resulting lift, drag and pitching moment polars are then included as part of the FLEXOP model for SHARPy, which include the two dimensional viscous effects.

With the fuselage and the viscous corrections from the airfoil polar data, we analyzed the FLEXOP aircraft with these enhanced modeling features. The results for the lift, drag polar and pitching moment coefficient are shown in Fig. 14. The lift curve sees minor differences across the different modeling features, all staying fairly close to the wing solved with the UVLM baseline. This is as expected given that, in the case with polar corrections, the thin FLEXOP airfoil has a lift curve slope close to $2 \pi$ at the set Reynolds number and that the fuselage is a nonlifting body. Therefore, we just observe curves with just the fuselage to produce slightly less lift, as a result of the reduced wing area, and a very minor reduction to the lift curve slope. The aircraft's pitching moment coefficient follows a similar trend, since it is a lift-dominated effect and we have seen how there is little impact on it arising from the fuselage or the airfoil data. Note that the pitching moment coefficient is computed about the aircraft's reference node in SHARPy (noted A) located at the wing-fuselage beam intersection.

On the other hand, the drag polar is the one that changes most with the contribution of the viscous, zero-lift drag from the airfoil polar, as this is otherwise neglected in the potential flow model of the UVLM. Immediately, we see this term shifting the aircraft drag curve, with a minimum drag coefficient of $C_{D, \min } \approx 0.009$ for all cases with the polar correction, as well as translating from approximately $-3^{\circ}$ to $-1^{\circ}$ the angle of attack at which the minimum drag occurs. In addition, the fuselage adds a small contribution pressure drag (inviscid) which increases with angle of attack. Of course, in a viscous simulation, the skin friction drag contribution of the fuselage would be significant given the large wetted area. However, it must be noted that this is an enhancement to the UVLM that would otherwise not even include the fuselage's contribution.

Table 2 Lift and moment curve slope and minimum drag of the FLEXOP aircraft under different modeling features.

\begin{tabular}{lccc}
\hline Modeling features & $\begin{array}{c}\text { Lift curve slope } \\
C_{L_{\alpha}}[/ \mathrm{rad}]\end{array}$ & $\begin{array}{c}\text { Minimum drag } \\
C_{D, \min }\end{array}$ & $\begin{array}{c}\text { Pitching moment slope } \\
C_{M_{\alpha}, \mathrm{A}}[/ \mathrm{rad}]\end{array}$ \\
\hline UVLM wing & 4.72 & 0.0000 & -3.58 \\
UVLM wing + fuselage & 4.60 & 0.0000 & -3.68 \\
Wing + polar correction & 4.81 & 0.0093 & -3.69 \\
Wing + fuselage + polar correction & 4.69 & 0.0088 & -3.79 \\
\hline
\end{tabular}

\section{Conclusion}

The present work has reported various improvements made to the open-source, nonlinear aeroelastic simulation environment SHARPy. These improvements aim to tackle some limitations of this environment by extending the capabilities of its aerodynamic solver. The aerodynamic solver is based on the UVLM which has been extended by 1) non-lifting body effects, 2) polar corrections and 3) a new wake discretization scheme.

The non-lifting body effects are captured by the linear source panel method which can be coupled by using superposition with the UVLM. Further, a special fuselage junction handling is implemented to avoid abruptly ending vortex panels, which would otherwise result in a non-physical local vortex shedding. First, the non-lifting body model has been verified with the analytical solution of potential flow over an ellipsoid. Second, the coupling of this model with the UVLM also demonstrated matching results with experimental data for a mid-wing and low-wing configuration. Third, we showed that for very flexible wings, the fuselage causes a lift increment that becomes non-negligible for high wing deformations.

The correction of the aerodynamic forces from the UVLM using the airfoil polar data enables a first approximation to viscous effects. In particular, zero-lift drag which would otherwise be neglected in the potential flow implementation enables a more accurate estimation of flight dynamic and aircraft performance characteristics. The correction of the lift 

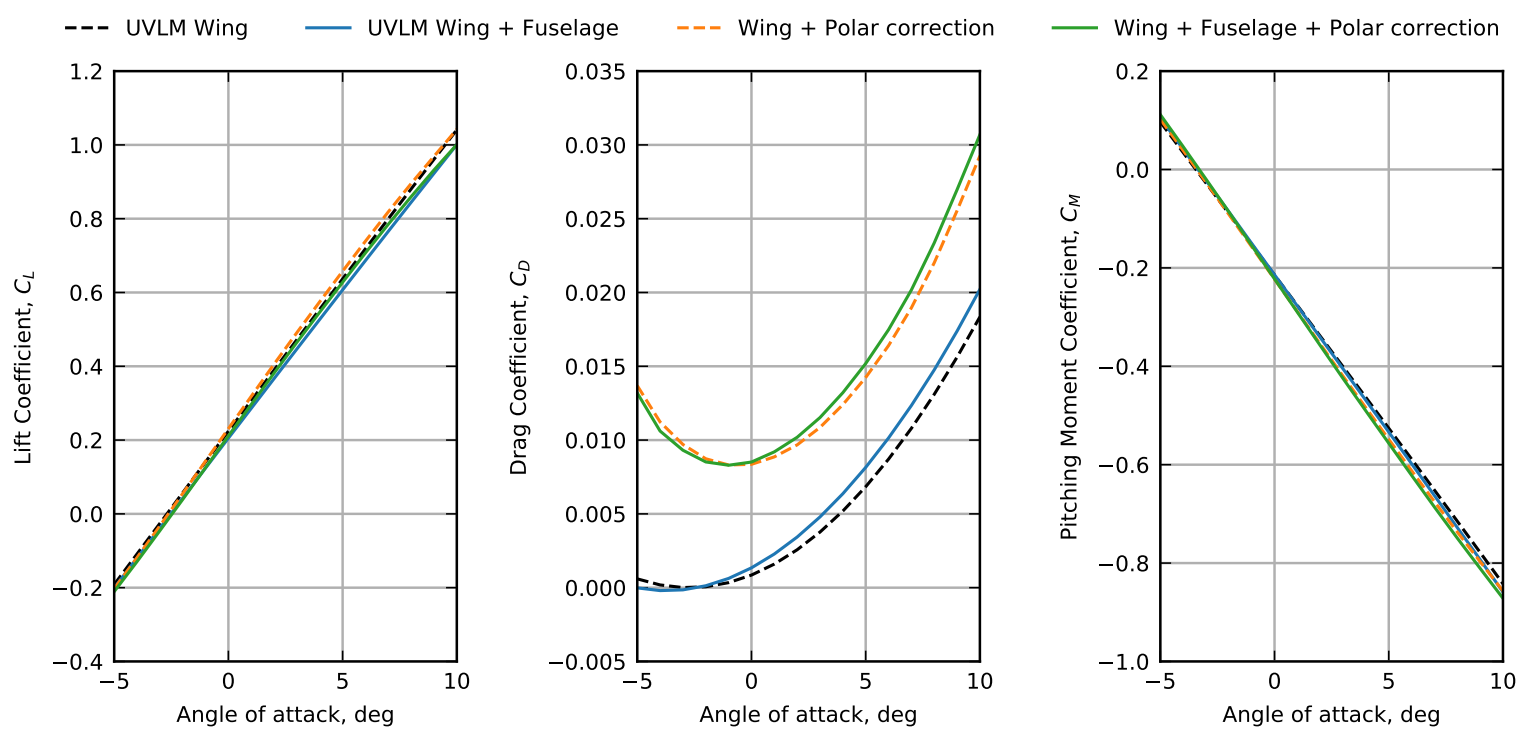

Fig. 14 FLEXOP aircraft lift, drag polar and moment curves under the different modeling enhancements

and pitching moment offers the possibility to explore regions at high angles of attack prior to the stall (for static cases) where the lift curve slope would depart the linear regime in a viscous flow.

In the new discretisation scheme, we exploit the fact that above a certain distance the unbound vortices in the wake have a negligible influence on the bound vortices on the wing. This allows us to greatly decrease the resolution of the wake far away from the wing and thus reduce the computational costs significantly. The method was verified by exposing an infinite, rigid wing to a Küssner gust. For this test case, the results match the analytical solution of this problem and no difference between the steady sized and variable discretized wake occur.

Finally, we used a preliminary model of the FLEXOP UAV newly implemented to SHARPy as a demonstrator model to apply our improvements on a flying aircraft. So far, static coupled simulations have been carried out. Our results indicate a good capture of the FLEXOP's structural behaviour. Further, we could see minor fuselage effects for the FLEXOP due to a lift drop near the junction. However, for significant effects on structural deformations as seen previously, the FLEXOP's fuselage is too narrow and achieved wing deformations to small.

By using the FLEXOP airfoil polar data to modify the UVLM-derived forces, we obtain first estimates for the vehicle's zero-lift drag. This is a key characteristic that will become crucial in time domain simulations focusing on flight dynamics, as longitudinal modes like the phugoid are greatly affected by it. In the case of static results for design and analysis of aircraft, knowing the aircraft's zero-lift drag permits more realistic estimations, for instance, of the thrust required to trim the vehicle as well as other aircraft performance characteristics, which can now also be explored from an aeroelastic perspective.

Work is currently underway to extend the coupling of the UVLM with the linear source-panel method for unsteady simulations. This unsteady coupling will be tested on aircraft gust responses, for which the wake discretization scheme presented here will be a key feature in reducing the expected high computational effort.

\section{Acknowledgment}

Stefanie Düssler's work has been funded by the COMAC-Imperial Research Centre for Wing Technology of Commercial Aircraft. Norberto Goizueta's research is made possible by a Ph.D. Scholarship from the Department of Aeronautics at Imperial College London, whose support is gratefully appreciated and acknowledged. The authors would like to thank Jurij Sodja from TU Delft for providing the FLEXOP structural information. 